\title{
CAMPO POLÍTICO, CAPITAL SOCIAL Y PARTICIPACIÓN: UN ANÁLISIS DE SUS DIVERSOS POSICIONAMIENTOS EN EL DEBATE DEL DESARROLLO
}

\author{
Emanuel Rodríguez Domínguez \\ zayolin@gmail.com \\ Escuela Nacional de Antropología e Historia \\ México
}

\section{RESUMEN}

El presente artículo propone una revisión crítica del concepto de capital social en los debates sobre desarrollo con el fin de revalorizar su utilidad metodológica en el análisis de la acción política. A partir de un recorrido conceptual por los diversos enfoques teóricos que definen esta categoría, se concluye que las investigaciones de los últimos años ligadas al desarrollo han promovido una visión «neutral» del capital social que pondera los elementos positivos del individuo como perteneciente a diversos entramados sociales. El artículo muestra por qué en los debates teóricos sobre desarrollo están ausentes el análisis y el diagnóstico de los aspectos negativos que pueden derivarse de la generación y reproducción de capital social entre los actores de un campo político.

Palabras clave: capital social, redes sociales, campo político, participación social, relaciones de poder, Robert Putnam, Pierre Bourdieu. 


\section{ABSTRACT}

This article proposes a critical revision of the concept of social capital within the development debates in order to reassess its methodological usefulness in the analysis of political action. Based on a conceptual review of different theoretical approaches defining this category, it is concluded that development-related research in recent years has promoted a «neutral» vision of social capital that ponders the positive elements that individual shave by belonging to various social grids. The article shows why the development debates lack a theoretical level with which to analyze and diagnose the negative aspects that the production and reproduction of capital social may have among the actors in the political arena.

Key words: social capital, social networks, politics, social participation, power relations, Robert Putnam, Pierre Bourdieu. 


\section{INTRODUCCIÓN}

En el campo de la antropología, los debates sobre el tema del desarrollo se han mostrado ambivalentes. ${ }^{1}$ No obstante, tal como establece Arturo Escobar, aunque la ecuación antropología-desarrollo se entiende y se aborda desde puntos de vista muy distintos, es posible distinguir dos grandes corrientes de pensamiento: aquélla que favorece un compromiso activo con las instituciones que fomentan el desarrollo en favor de los pobres con el objetivo de transformar la práctica del desarrollo desde dentro - la antropología para el desarrollo_-; y aquélla que prescribe el distanciamiento y la crítica radical del desarrollo institucionalizado — la antropología del desarrollo - (Escobar 1997).

En el marco de este debate, el discurso del desarrollo puso en un primer plano, en sus estrategias y proyectos aplicativos, la atención a factores sociales y culturales.

Ejemplo de ello fue el giro que efectuaron diversas instituciones para el desarrollo en las últimas décadas del siglo XX, principalmente agencias internacionales como el Banco Mundial, la Agencia para el Desarrollo Internacional de Estados Unidos (USAID), y algunas oficinas técnicas de las Naciones Unidas, al adoptar una política de programas focalizados donde se capacitaba a la población objetivo para que colaborara en la solución de una problemática particular (generalmente sobre los temas de pobreza y marginalidad). Así, los diseñadores de estos programas aceptaron que los pobres debían participar activamente en las estrategias de desarrollo si se pretendía alcanzar algún resultado positivo (Cernea 1985, citado en Escobar 1997).

Al proponerse superar una visión de la intervención de «arriba hacia abajo», los proyectos de desarrollo pretendían reforzar su impacto social y ser culturalmente adecuados, por lo que empezaron a tomar en consideración a los beneficiarios directos de un modo sustancial. Ante tal situación, uno de los conceptos que empezó a figurar en los debates del desarrollo fue la noción de «capital social», pues dicho término aludía directamente a la serie de 
recursos y componentes adquiridos como consecuencia de las relaciones de una persona con otras, así como a la participación de los actores sociales en organizaciones formales y en las arenas políticas del ámbito local. Paulatinamente, la noción de capital social empezó a ser empleada como un indicador crucial para la puesta en marcha de los programas de desarrollo, al grado que llegó a considerarse una «herramienta neutral y útil», pues fomentaba la participación social en las estrategias que pretendían mejorar el nivel de vida de un grupo o colectividad (Durston 2000, 2001, Klisberg 2000).

En sentido contrario, las críticas expuestas por la antropología del desarrollo empezarían a alertarnos sobre una de las grandes paradojas de las políticas y acciones aplicativas implementadas por las agencias internacionales: la poca relación existente entre el discurso del desarrollo y la práctica empírica del mismo (Gow 2002). Esto originó cuestionamientos en el sentido de cómo, en algunos casos, las cualidades que se le asignaban a la categoría de capital social en el diseño de un proyecto aplicativo eran constructos que conformaban el imaginario teórico sobre el posible fomento de habilidades sociales para el mejoramiento del nivel de vida de los sujetos, cuyo sustento estaba más en los ideales e intencionalidades del creador de la propuesta que en la evidencia empírica. Por tanto, era indispensable reconocer que existían limitaciones en la conceptualización del capital social (Putzel 1997, Harriss y De Renzio 1997), las cuales hacían más complejo el debate de la participación de la sociedad en las estrategias o programas de desarrollo.

Precisamente, este ensayo retoma la discusión anterior y examina cómo los usos del término capital social dentro de los debates del desarrollo han generado una amplia gama de interpretaciones que requieren de una precisión conceptual para que dicha categoría no pierda su utilidad como recursos heurístico. En primer lugar, se apuesta por hacer un uso restringido y acotado de este término en las estrategias de desarrollo con el afán de mantener su utilidad metodológica, pues dicha cualidad contribuye en gran medida a establecer valiosos puentes analíticos entre los elementos socioculturales que aborda la antropología y las variables de estudio clásicas de la ciencia económica. En segundo lugar, 
se destaca que los proyectos para el desarrollo siempre serán implementados en un contexto sociocultural entrecruzado por múltiples variables (constantes conflictos, procesos de intermediación, evaluaciones pragmáticas de los actores sociales, etc.), las cuales impactan directamente en las relaciones sociales. Esto nos ayudaría a entender, por un lado, por qué en los proyectos de desarrollo las relaciones sociales no siempre circulan por sendas armónicas o sobre escenarios participativos estrictamente horizontales. Por otro, también nos permite entender por qué el fomento de capital social en una comunidad no necesariamente servirá para mejorar el nivel de vida de sus miembros, pues en algunos casos puede servir también para fomentar las desigualdades que existen en todo entramado social.

\section{CAPITAL SOCIAL: UNA MIRADA A LA DISCUSIÓN CONCEPTUAL}

El análisis de los vínculos entre los actores sociales representa el nodo principal de las ciencias de lo social. La preocupación por la solidaridad social como germen fundamental de la cooperación y la cohesión social guió los primeros estudios sociológicos. En este sentido, antes de iniciar una discusión conceptual sobre lo que hoy se entiende como capital social, es conveniente retomar la advertencia que hace Alejandro Portes al considerar que el conjunto de procesos abarcados por el término no son nuevos y han sido estudiados a lo largo de la historia bajo otras denominaciones (1999: 262). Sin embargo, no hay que confundir la existencia del fenómeno con el surgimiento de categorías analíticas más atractivas a nivel teórico-metodológico, las cuales nos permiten realizar una nueva lectura de las interacciones sociales a partir de puntos como el contexto social donde se desarrollan los sujetos sociales, la intencionalidad de sus acciones y sus procesos de significación cultural.

Para analizar el desarrollo del concepto, tomaré como punto de partida el trabajo de la urbanista Jane Jacobs (1961), The Death and Life of Great American Cities. En esta obra, Jacobs analizó las relaciones humanas de las ciudades y los grupos sociales que las habitan 
para determinar que una densa red de relaciones entre los actores sociales de las urbes incide de manera positiva sobre la estructura de la sociedad en general (Marrero 2006).

Cabe destacar que, en la década de los años setenta, la aplicación instrumental de las redes sociales fue un elemento ampliamente utilizado por los investigadores de la Escuela de Manchester, encabezados por Max Gluckman y J. Clyde Mitchell. Bajo este enfoque, destacan los trabajos de Jeremy Boissevain, Albert Trouwborst, Bruce Kapferer y Adrian C. Mayer, quienes utilizaron el término «inversiones» para obtener un mayor número de relaciones sociales (Molina 2001: 51). En el campo de la sociología económica surgieron algunas investigaciones, como las de Mark Granovetter (1973) y Glenn Loury (1977), que hacían referencia al valor agregado de las redes sociales para incidir positivamente en el nivel de vida de los actores sociales o en el desarrollo de su organización social. ${ }^{2}$

Si bien estos aportes metodológicos son referentes importantes para la discusión que nos atañe, es necesario retomar la primera formulación del concepto y el primer análisis sociológico sistemático contemporáneo que consideró dentro de su estudio al capital social, ${ }^{3}$ desarrollados por Pierre Bourdieu en su teoría de los campos sociales. Bourdieu definió el concepto de la siguiente manera: «El capital social está constituido por la totalidad de los recursos potenciales y actuales asociados a la posesión de una red duradera de relaciones más o menos institucionalizadas de conocimiento y reconocimiento mutuos. Expresado de otra forma, se trata aquí de la totalidad de recursos basados en la pertenencia a un grupo» (Bourdieu 1985: 148).

Enmarcado en este análisis, se considera el capital social como una construcción social atravesada tanto por las relaciones sociales objetivas, entendidas como ámbitos de acción (los campos sociales), como por las estructuras subjetivas o incorporadas (el habitus). Por ello, la construcción deliberada, las estrategias de inversión y el uso que hacen los actores sociales de redes duraderas de relaciones tienen un vínculo directo con las estructuras y los procesos que reproducen el poder y el mismo capital. Por lo anterior, el debate no sólo gira 
en torno a la identificación del acceso, monto y calidad de las relaciones, sino que también busca dar cuenta de las desigualdades (económicas, sociales y culturales) entre individuos que tienen un capital social similar. ${ }^{4}$

James Coleman contribuyó significativamente al debate sobre capital social desde una perspectiva económica que combina los procesos de elección racional realizados por un individuo y las teorías del sistema social. Este autor sostiene que el capital social no es una sola entidad sino «una diversidad de entidades con dos elementos en común: todos consisten en algún aspecto de la estructura social y facilitan ciertas acciones de los actores dentro de la estructura» (Coleman 1990: 302, citado en Forni, Siles y Barreiro 2004: 3).

Además, a un nivel instrumental explica que: «La función identificada por el concepto de capital social es el valor de esos aspectos de la estructura social que los actores pueden usar como recursos para la realización de sus intereses» (Coleman 1990: 305, citado en Forni, Siles y Barreiro, 2004: 3).

Bajo el modelo de Coleman, el capital social se concibe como una serie de recursos que los actores valoran y obtienen dentro de una estructura social al relacionarse entre ellos y que les sirven para lograr determinados fines o metas. ${ }^{5}$ En este sentido, los recursos para alcanzar un objetivo se ponen en acción cuando existen intercambios de favores entre individuos de una misma estructura social. Cabe destacar que los intercambios giran en torno a la reciprocidad «obligada», pero guiada por el interés personal de satisfacer ciertas necesidades y por el sistema de normas y sanciones compartidas (dentro de las cuales se agrupan desde las obligaciones y expectativas, hasta las normas y sanciones afectivas y de autoridad). Las críticas al modelo de Coleman se han enfocado en la centralidad que otorga al cálculo racional de los actores en las transacciones sociales, al ser guiados únicamente por su interés personal. Sin embargo, vale la pena resaltar su aporte conceptual, pues por medio de la noción de capital social este autor intentó tender un puente analítico entre los elementos micro y macro de la estructura social, al lograr, en primer lugar, «introducir la 
racionalidad individual en el plano de la acción, y en segundo lugar, mantener firme el contexto de la acción: la organización social» (Millán y Gordon 2004: 715).

Retomando la discusión de Coleman y guiado por la lente del neoinstitucionalismo económico, Robert Putnam desarrolló uno de los aportes más fructíferos al debate contemporáneo sobre capital social, puesto que, en gran medida, esta nueva conceptualización popularizó su uso como elemento fundamental dentro de los programas de desarrollo impulsados por agencias internacionales y organizaciones de la sociedad civil. La obra de Putnam (1993) propone un giro conceptual y metodológico donde el capital social se convierte en un componente comunitario y ya no en un elemento individual. En esta propuesta, las redes sociales son un canal mediante el cual sus integrantes, a través de la creación de capital social, pueden satisfacer necesidades individuales o colectivas. De acuerdo con este autor, las redes de relaciones son un elemento subjetivo compuesto por valores y actitudes que determinan la forma como ciertos actores se asocian con otros apoyándose en la confianza social, las pautas de reciprocidad y los valores de cooperación compartidos.

Desde este enfoque, el capital social es un atributo que proporcionan las redes sociales a los individuos, generándose así una visión comunitaria del concepto. Retomando a Putnam (1993), el capital social se puede identificar con la participación de los actores en organizaciones locales, clubes y asociaciones, así como en grupos cívicos. De esta manera, el capital social se refiere a las características de la organización social, como por ejemplo redes, normas y confianza, que facilitan la cooperación y la coordinación en beneficio mutuo. Éste es el motivo por el cual se convierte en un elemento vital para el desarrollo económico de los grupos, así como para su partición política. A pesar de que el estudio de Putnam considera la existencia de procesos de asociacionismo horizontales y verticales, su concepto de capital social alude directamente a las comunidades donde predominan las relaciones del primer tipo y a los efectos positivos (confianza, reciprocidad y compromiso cívico) de su densificación e institucionalización, cuyos beneficios económicos y políticos 
son el abaratamiento de los costos de transacción y la disminución de la incertidumbre al cooperar y participar en asuntos comunes.

Las tres posturas hasta aquí presentadas son un referente fundamental para analizar el papel del capital social en las estrategias de desarrollo. ${ }^{6}$ No obstante, es conveniente señalar los elementos que hacen que estas visiones no sean complementarias:

1) Por un lado, se encuentran los enfoques del capital social que consideran en su marco de análisis las desigualdades y el poder $\mathrm{y}$, por lo tanto, sus efectos positivos $\mathrm{y}$ negativos, así como las condiciones estructurales que lo generan.

2) Por otro, están las conceptualizaciones de capital social que ponderan los elementos positivos de las redes de relaciones y su disponibilidad para movilizar recursos en la búsqueda de un fin propio o colectivo.

Actualmente, en el debate sobre el desarrollo predomina la segunda posición, sobre todo en las agencias y organismos enfocados en este tema. El concepto ha cobrado relevancia por ser un indicador crucial para el éxito o fracaso de un proyecto aplicativo al grado de que, en el afán de afinar su utilidad analítica, se han generado una serie de clasificaciones que distinguen entre formas, tipos, alcances, ámbitos, niveles, etc. ${ }^{7}$

A pesar de sus limitantes y su fácil cuestionamiento, las múltiples aplicaciones del concepto de capital social en el debate del desarrollo no deben descartarse de facto, pues proporcionan elementos heurísticos que arrojan indicadores sugerentes para la definición de estrategias de acción que tienen como fin mejorar el nivel de vida de un grupo o colectividad. Sin embargo, para que los indicadores de capital social de una comunidad sean representativos y sirvan de base para una estrategia de desarrollo local, éstos deben de surgir de un tratamiento sistemático que analice las relaciones sociales de un grupo o colectividad; a saber: a) los poseedores de capital social (quienes hacen reclamos y establecen las relaciones sociales), b) las fuentes de capital social (quienes aceptan esas 
demandas y elaboran las agendas de desarrollo) y c) los recursos disponibles en los contextos donde se desarrollan los actores (Portes 1999: 247).

Cabe destacar que no se trata de descalificar o poner a competir las perspectivas antes mencionadas, pues el gran aporte de todas ellas radica en que abrieron el debate sobre el capital social en las estrategias del desarrollo. Además, dichas perspectivas han buscado dar un respaldo teórico-metodológico desde diferentes disciplinas a un proceso de la realidad social en constate reconstrucción: la densificación de las relaciones sociales para fines colectivos. Precisamente este ensayo busca contribuir a dicho debate estableciendo que a la noción de capital social no le vendría mal retomar las bases iniciales de su concepción, es decir, la teoría de los campos sociales, pues consideramos que de esa forma se podrían solventar las paradojas existentes entre los discursos y las prácticas empíricas de un proyecto aplicativo.

Por ello, en las siguientes líneas discutimos si la conceptualización de capital social requiere un acotamiento analítico para potenciar su utilidad heurística. Nuestro punto de partida establece que algunas interpretaciones y aplicaciones del concepto en las estrategias del desarrollo no han considerado los conflictos ni la heterogeneidad de la sociedad donde se intenta establecer un proyecto para incentivar la creación de capital social (Natal 2010), con lo cual han cambiado radicalmente el sentido de este término y únicamente han fomentado la confusión en sus usos y alcances dentro de cualquier programa o acción destinado a mejorar el nivel de vida de una colectividad o grupo social.

No obstante, desde nuestro punto de vista, el análisis del capital social bajo una óptica procesual puede contribuir a superar su concepción «neutral»o «idealizada»al incorporar el análisis del conflicto y las relaciones de poder a la densificación de las relaciones sociales. Esto nos permitiría establecer un puente entre los aspectos positivos y negativos que presenta la creación de capital social en un grupo o colectividad y con ello tener un panorama más completo del papel que juega dicho término en los proyectos de desarrollo. 


\section{LOS CLAROSCUROS DEL CAPITAL SOCIAL EN LOS DEBATES CONTEMPORÁNEOS DEL DESARROLLO}

Es innegable que actualmente el capital social es un recurso analítico que se ha posicionado como uno de los grandes tópicos en las ciencias sociales al promover un enriquecimiento recíproco entre conceptualización, investigación y práctica social. Ello se debe a que, a diferencia de otros, así como se teoriza y debate a nivel conceptual en trabajos explicativos o interpretativos con relación a temas como la pobreza, las políticas públicas y la participación ciudadana, ${ }^{8}$ también se aplica en un sinnúmero de proyectos promovidos por agencias internacionales y organizaciones de la sociedad civil. ${ }^{9}$ Si bien el desarrollo del concepto puede criticarse por haber ocupado un lugar central en la moda desarrollista, al grado de ser considerado en el seno del Banco Mundial como el «eslabón perdido» que podría solucionar la pobreza, ${ }^{10}$ esto no minimiza los aspectos que contribuyen a la imagen positiva del concepto y sus efectos prácticos en el involucramiento de las comunidades en las agendas del desarrollo.

El capital social debe entenderse como la conceptualización de una práctica social que se presenta en grupos no homogéneos, lo que hace que sus efectos $y$ funciones se diversifiquen y dependan en gran medida del contexto donde se desarrollan las relaciones sociales. Por ello, no se puede negar que este concepto ha establecido una serie de puentes interdisciplinarios entre las ciencias sociales con el afán de captar la complejidad de los procesos de desarrollo y se ha considerado no sólo en términos económicos, sino también desde el punto de vista de los elementos socioculturales. Bajo este marco de interpretación, Bernardo Kliksberg enfatizó la importancia de tener una visión integral para analizar el capital social en los proyectos de desarrollo. Al estudiar algunas experiencias exitosas ${ }^{11}$ que promovieron la movilización de capital social y cultural comunitario, así como un diseño de gestión abierto y democrático por parte de las autoridades y de las agencias internacionales, dicho autor concluyó que los elementos culturales de una colectividad, vinculados a los factores sociales y económicos de sus contextos, pueden generar un instrumento formidable 
de progreso económico: el desarrollo del capital social (Kliksberg 2000: 22).

Estudios como los de Kliksberg han resultado reveladores en el tema del capital social al demostrar cómo la consideración y valoración de los aspectos socioculturales de las redes de relaciones permiten fomentar acciones de respeto hacia formas específicas de identidad y pertenencia étnica, tales como tradiciones, memoria social, prácticas religiosas y formas de producción y reciprocidad comunitaria, entre otros «satisfactores socioemocionales» (Durston 2000, citado en Arriagada 2003: 25). Esto es de suma importancia para avanzar en la trasformación de las relaciones de reconocimiento y aceptación de la diversidad sociocultural, además de que el actor y su conocimiento local son la base para el diseño de aquellos programas aplicativos que le permiten obtener beneficios individuales y colectivos. De esta forma, es posible reforzar la organización social y fomentar estrategias para el desarrollo desde la propia perspectiva de los actores.

La reconfiguración de la idea de desarrollo desde la óptica de las comunidades refleja un ejercicio del derecho de sus habitantes a decidir sobre su propio destino por medio de una plena y activa participación democrática. Así, la participación ciudadana no sólo debe ser entendida como el ejercicio de un derecho democrático, sino que debe ser pensada también en función de distintos grados de involucramiento de los actores en la solución de los problemas que les afectan (Miranda 2003). Esto permitiría fomentar la integración social y la conformación de redes sociales más densas que posibilitarían el empoderamiento de organizaciones comunitarias, lo que a su vez promovería un fortalecimiento institucional que, en teoría, mejoraría la atención de las demandas que elevan las comunidades al Estado. Sin embargo, la solución no radica únicamente en fomentar los procesos participativos de un grupo o colectividad, ya que también existen algunas relaciones institucionales viciadas o corrompidas en su interior. Además, el empoderamiento de un sector supone la pérdida de poder o, al menos, del interés del otro sector para compartir el poder de su esfera política y en verdad generar un proyecto colectivo que beneficie a los diversos sectores de la sociedad. $^{12}$ 
En lo hasta ahora expuesto podemos observar la utilidad operativa que se le atribuye al concepto de capital social. Por un lado, su análisis permite identificar los activos o recursos de algunos sectores que no están siendo plenamente utilizados para buscar mejoras en sus vidas cotidianas, lo que se relaciona directamente con una temática contemporánea importante: los procesos de desigualdad existentes en una sociedad. Por otro, no se puede ignorar el aporte que arrojan los estudios sobre capital social en las acciones para el desarrollo en cuanto a la creación de indicadores, sobre todo aquéllos que, desde una perspectiva sistemática e integral, analizan multidimensionalmente las condiciones de pobreza de una comunidad y las posibles estrategias para superarla, a fin de dar luz a campos de oportunidad que deben ser atendidos a través del diseño pertinente, eficaz, relevante y, sobre todo, participativo de programas de acción aplicados por una macro red interorganizacional que vincularía a la ciudadanía en general, las organizaciones de la sociedad civil, las agencias de desarrollo y las estructuras estatales.

Ahora bien, hasta este momento hemos presentado una revisión de los factores positivos del concepto de capital social y su aplicación analítica. Sin embargo, para no formarnos una idea sesgada, cabe añadir algunas reflexiones generales que describen los efectos no tan positivos del capital social en las estrategias de desarrollo y en las investigaciones que lo evalúan como un «motor» de participación horizontal en todas las sociedades. Las principales desventajas del término van asociadas a la conceptualización teórica y a la clasificación taxonómica de las propuestas aplicativas, que han simplificado la realidad asumiendo la «naturalidad» de ciertas relaciones sociales que implican conflicto y desigualdad (Montaño 2003: 69).

Generalmente, los estudios que abordan los aportes del capital social en un proyecto de desarrollo no contextualizan a los sujetos en sus entornos de acción (el campo social). En consecuencia, dejan fuera del análisis elementos importantes que tienen que ver con las relaciones de poder que se recrean al poner en práctica una estrategia de desarrollo. 
Esto se debe a que, generalmente, las estrategias para el desarrollo presuponen que sus proyectos tienen como eje de interacción una serie de redes de relaciones enmarcadas en una comunidad armónica idealizada que no puede ser alterada. Dicha idea minimiza, y en cierto sentido olvida, que el capital social, antes que nada, es una práctica influida por contextos socioculturales cruzados por conflictos, procesos de intermediación e intereses grupales y colectivos derivados principalmente de las diferencias culturales, ideológicas y económicas que existen en los entornos locales (Natal 2010).

Uno de los errores más notorios del enfoque «neutro»-que deja fuera las relaciones de poder- ha sido la omisión de las diferencias de acceso a las redes de relaciones y a los recursos que dichos entornos proveen a hombres y a mujeres. De esta manera, se cuestiona la utilidad del capital social en los proyectos del desarrollo que no consideran el entorno de aplicación, ya que proporcionan una visión parcial de la complejidad social. Esto no niega que existe una gran capacidad analítica para dar cuenta de los procesos de articulación y empoderamiento de algunos grupos sociales y colectividades en contextos marginales, lo cual es de gran utilidad en el diseño de una estrategia de desarrollo. Sin embargo, retomando una experiencia personal de investigación, ${ }^{13}$ también se corre el riesgo de considerar simétrico el acceso a los recursos de capital social entre todos los actores sociales, siendo que en algunos casos las redes pueden servir como medios para el mantenimiento de las desigualdades, por ejemplo, las de género (Anderson 2008).

Los enfoques «neutrales» del uso del capital social en el fomento a la participación dentro de los programas de desarrollo son también criticados por omitir el sistema político cuando ponderan el reforzamiento del sentido colectivo de una comunidad para vincularse con las estructuras gubernamentales o con las agencias de desarrollo (Durston 2000; 2001). Por lo general, estos estudios no toman en cuenta que en algunos casos la corrupción y diversos actos de coyotaje generados desde instancias estatales merman la credibilidad del desempeño gubernamental y, por ende, la participación de los actores sociales en los proyectos de desarrollo. Este tipo de procesos nos advierten que en las redes sociales no 
sólo se realizan acciones que promueven el asociacionismo colectivo horizontal o vertical, la reciprocidad y la confianza generalizada, un mayor nivel de compromiso cívico y una participación democrática, sino que también existen vínculos y relaciones multilaterales (tanto al interior de las comunidades como entre las agencias de desarrollo y las instancias estatales), en los cuales es común encontrar actores que operan como free riders ${ }^{14}$ al perseguir un beneficio individual más que colectivo (Levi 1996).

Por lo anterior, algunas críticas antropológicas al desarrollo se pueden hacer extensivas a las investigaciones basadas en el capital social, pues dichos estudios dejan fuera el análisis del discurso de poder que representan los programas aplicativos de las agencias internacionales y las organizaciones de la sociedad civil (Harriss y de Renzio 1997). En este sentido, lo único que han logrado los programas de desarrollo es legitimar el discurso que establece que las acciones de intervención son un proceso «neutral» que promueve la participación colectiva de las comunidades en el mejoramiento de sus niveles de vida, con lo que se pretende convertir los conflictos políticos derivados de un proyecto aplicativo en una suave y armoniosa construcción de consensos entre las poblaciones objetivo. Sin embargo, hay que tomar en cuenta que, si bien por medio del capital social se pueden establecer coaliciones (asociaciones verticales), también dentro de éstas se pueden conformar facciones, lo cual nuevamente lleva el análisis a la esfera del poder (Bahamondes 2003). Por ello, es importante destacar que, si partimos de adjudicar un sentido positivo a la participación ciudadana en los programas de desarrollo sin considerar el contexto donde se lleva a cabo la acción política, se crea un escenario ideal donde las relaciones son armónicas. Así, estamos actuando a partir de una concepción de capital social que tiende a ser normativa y que busca ordenar lo social, en lugar de ser una herramienta descriptiva y analítica.

Por otro lado, se ha dado por hecho que las redes de reciprocidad y confianza generalizada son un factor «natural» en los procesos de asociacionismo horizontal e incluso suelen vincularse directamente con los sectores sociales de bajos ingresos o rurales, lo que 
nuevamente presupone colectivos homogéneos y estáticos. Este supuesto fue una de las principales críticas que se hicieron a los estudios de redes sociales que intentaron explicar las estrategias de sobrevivencia de los sectores marginados (Lomnitz 1994). Sin embargo, incluso en estos trabajos se advierte la existencia de relaciones patrón/cliente, que se caracterizan por ser relaciones asimétricas dentro de una red social.

Esta precisión nos lleva a plantear un problema de acaparamiento en los análisis de capital social, con lo que su aplicación podría derivar en un clientelismo instrumental utilizado por líderes locales (brokers), ${ }^{15}$ autoridades gubernamentales y otros actores implicados en los programas de desarrollo.

Los ejemplos anteriores muestran cómo el capital social no siempre tiende a incentivar una participación horizontal en las estrategias de desarrollo que pretenden impactar sobre el nivel de vida de los actores sociales de un entorno local. En este sentido, siguiendo a James Putzel, debemos tomar en cuenta que las redes de relaciones de los actores involucrados en los procesos de desarrollo también pueden generar un capital social «con externalidades negativas» o un «capital social deficitario». Este «otro lado» del capital social puede fomentar relaciones de discriminación, explotación, corrupción y acaparamiento de recursos por parte de una facción, así como el establecimiento de sistemas autoritarios por parte de un grupo hegemónico, la aparición de conflictos intergrupales y el aprovechamiento injusto de los esfuerzos colectivos (Putzel 1997). La aparición de este tipo de prácticas nos invita a pensar en la importancia del conflicto en las redes sociales, pues en cierta medida uno de los objetivos implícitos en la cooperación colectiva es controlar la mayor cantidad de recursos para poder realizar agendas particulares, lo que implica que otros grupos no accedan a los recursos en disputa o se subordinen a los intereses del grupo que mantiene el control de los mismos. Así, el capital social puede contribuir a mantener las desigualdades y la exclusión en grupos socioculturalmente diferenciados (Harriss y de Renzio, 1997). 
En lo presentado hasta este momento se ha dibujado un panorama general de lo que a mi juicio representa los claroscuros del capital social en el debate del desarrollo. Tenerlos en cuenta en nuestras investigaciones implica también una reflexión para evitar ponderarlos en exceso o, en sentido contrario, omitirlos totalmente del análisis. Ello nos ayudaría a corregir las visiones sesgadas y enfocadas a una o dos variables (regularmente económicas y culturales). Sin embargo, aún nos falta explicar en qué medida el contexto donde se desarrollan los sujetos sociales condiciona la creación de capital social y los fines del mismo. Esta contextualización, como veremos a continuación, inscribe el capital social inmediatamente en un campo dinámico de relaciones sociales donde el actor social encuentra un sinnúmero de posibilidades de acción, pero la misma cantidad de restricciones.

\section{HACIA UNA CONTEXTUALIZACIÓN Y DELIMITACIÓN DEL CAPITAL SOCIAL: LOS LÍMITES DE LA ACCIÓN POLÍTICA EN LOS CAMPOS SOCIALES}

Hasta aquí hemos resaltado el intenso debate que considera el capital social como una teoría, un concepto o un recurso para ser aplicado a la investigación de problemas concretos. Esto, por un lado, nos impone el reto de precisar qué es el capital social: un elemento existente que puede ser abstraído, una metáfora para analizar la realidad, un cúmulo de relaciones medibles, entre otras acepciones que se le atribuyen al concepto. Por otro, nos deja la tarea de generar una contextualización de dicho elemento dentro de la noción de campo social a fin de superar los límites analíticos que se le imputan por considerar las redes de relaciones como elementos estáticos, instrumentales, neutrales e independientes de otras variables y procesos del entorno social.

En primer lugar, el capital social es una herramienta que nos ayuda a representar la realidad social. No obstante, retomando las precisiones que contemplan los estudios de redes sociales (Molina 2001) ${ }^{16}$ hay que tomar en cuenta que la realidad no está conformada por redes (como elementos tangibles), sino que representamos dichas prácticas sociales usando 
una categoría o concepto en un sentido metafórico, lo cual nos remite a la existencia de un marco interpretativo que le otorga sentido y poder explicativo. Por ello, quizá la mayoría de los debates teóricos sobre capital social han encasillado su análisis y aplicación dentro de una visión racionalista que ha dejado fuera las diferenciaciones sociales, culturales y económicas existentes entre las comunidades donde se implementan las estrategias de desarrollo. En consecuencia, estos estudios sostienen una visión limitada del capital social, pues lo consideran un elemento neutral que generalmente promueve beneficios colectivos.

Para superar estas visiones reduccionistas del capital social, propongo contextualizar nuevamente su estudio dentro de la teoría de los campos sociales de Pierre Bourdieu (1990) y del procesualismo político de la Escuela de Manchester. ${ }^{17}$ El regreso del capital social a su nicho teórico nos permite analizar las relaciones entre los sujetos como parte de una esfera social que tiene lógicas de estructuración y de dominación propias, con un alto grado de diferenciación y complejidad. Bajo este enfoque, el campo social debe entenderse como un sistema de relaciones de fuerza y de sentido entre diversos grupos, pues dichas colectividades tienen por objetivo generar o reproducir su capital cultural, social y económico. De ahí que la constitución de un campo dependa de la existencia de un capital común y de la lucha por su apropiación. ${ }^{18}$

El estudio de capital social contextualizado en la teoría de los campos nos permite analizar dinámicamente las lógicas interna y externa de las redes sociales con el fin de ver cómo se producen dentro de un entorno específico, para posteriormente analizar operativamente su formalización en las prácticas cotidianas a través de su reproducción.

Esta aproximación inicial puede ser útil para determinar qué tan desiguales son los beneficios para los integrantes de una red social que tienen el mismo capital social, factor que desde mi punto de vista debe considerar cualquier programa de desarrollo que se diga inclusivo de la visión local. Así, los procesos de poder y reproducción del mismo, dentro y fuera de las redes de relaciones, tienen que ser considerados en el análisis del capital social, 
pues entender cómo se han ido estructurando las posiciones y jerarquías de los actores sociales dentro del campo político sería el primer paso para reconfigurar la desigualdad de posiciones en los entornos locales.

Ahora bien, no sólo Pierre Bourdieu ha puesto atención al análisis multidimensional de las relaciones sociales por medio de campos de acción, también la perspectiva procesualista, con un enfoque diferente, nos advierte que la sociedad puede ser entendida como un conjunto de campos con muchas dimensiones que pueden estar vagamente integradas o ser virtualmente independientes una de la otra (Swartz, et al. 1966: 102). Para el procesualismo político, una de las dimensiones más importantes donde se desarrollan los sujetos es el campo político, ya que dicho entramado de relaciones nos permite visibilizar de manera integral las disputas por el control de los recursos de la sociedad - el poder-, las acciones específicas del gobierno o de una agencia de desarrollo — la política- y el juego cotidiano de agentes por espacios de poder e influencia —lo político— (Rodríguez 2010).

El procesualismo nos permite establecer que la implementación de un proyecto de desarrollo en una comunidad siempre genera una serie de arenas políticas en las que los actores sociales, individuales y colectivos interactúan buscando persuadir a otros actores, dentro y fuera de sus grupos de referencia, sobre la preponderancia de sus valores. De ese modo, intentan construir una red más fuerte (con más capital social) y direccionar los proyectos del desarrollo a favor de agendas particulares (Natal 2010). En este sentido, las acciones del desarrollo siempre van a estar marcadas por aquellos procesos políticos que tienen que ver con la disputa de recursos, lo que provoca que los proyectos de desarrollo no respondan a las necesidades o intereses de todos los miembros de una sociedad y se generen conflictos propios de un entorno social fragmentado y con gran diversidad sociocultural.

Esto implica que los actores involucrados en las estrategias de desarrollo no siempre reflejen una total oposición a las acciones emprendidas ni expresen tampoco un absoluto 
acuerdo.

Por ello, se considera importante diagnosticar los entornos de conflicto en los procesos de implementación y operación de cualquier proyecto aplicativo a fin de canalizarlos hacia un entretejido de relaciones que fomente el debate y la participación de los diversos sectores involucrados.

En conclusión, el análisis del papel del capital social en las estrategias del desarrollo desde la óptica de los campos sociales nos permite ver cómo las relaciones sociales no ocurren en contextos neutrales o entornos armónicos, sino que constituyen una expresión de valores e intereses condicionados por factores históricos, percepciones interiorizadas de los actores sociales, relaciones asimétricas de poder, así como por una constante lucha social por la asignación de significado a las relaciones sociales. En este sentido, podríamos establecer que la participación de los sujetos en las estrategias de desarrollo no tiene una meta predefinida, sino que más bien se mueve en un continuиm entre el conflicto, que promueve la existencia de capital social negativo, y el consenso, que genera capital social positivo.

\section{CONCLUSIONES}

A lo largo de este trabajo se han presentado diversas posturas sobre el estudio y aplicación del capital social en los programas de desarrollo. Por un lado, esta aproximación intenta dejar atrás la idea de que la densificación de las relaciones sociales de un grupo o colectividad se realiza en contextos armónicos que promueven escenarios de participación horizontales en las estrategias que pretenden mejorar el nivel de vida de un grupo o colectividad. Por otro, la revisión tiene como objetivo generar una matriz explicativa que retome el concepto de campo social, entendido como el entorno de acción donde los sujetos pueden ampliar el alcance de sus redes sociales para contextualizar la producción, reproducción y apropiación del capital social. 
Al recuperar la noción de campo social en el análisis de las estrategias de desarrollo, rescatamos también la utilidad metodológica del concepto de capital social en relación con: 1) el contexto donde se implementa el proyecto aplicativo, 2) las intencionalidades de los actores involucrados — ciudadanía, estructuras gubernamentales, agencias internacionales y organizaciones de la sociedad civil- y 3) los procesos culturales que giran en torno a los programas de desarrollo. Estos tres elementos nos permiten establecer que, en la implementación de este tipo de estrategias, no siempre vamos a encontrar escenarios donde predominen el asociacionismo, la reciprocidad o la participación horizontal, sino que también encontraremos actores que ponderen y promuevan procesos de intermediación, conflictos por los recursos, negociaciones pragmáticas, así como la implementación de esquemas de participación antidemocráticos.

Ubicar los múltiples sentidos que puede tener la creación, reproducción y disputa del capital social en un proyecto de desarrollo es fundamental en la implementación y operación de cualquier proyecto aplicativo, pues de esta manera podremos saber con mayor precisión si la participación de los actores sociales está fluyendo por sendas que promueven el beneficio colectivo de una comunidad, o si por el contrario el proyecto está promoviendo una mayor desigualdad y fragmentación social en la población objetivo. Así, el propósito de este documento no es demeritar las valoraciones positivas que tiene la noción de capital social en el debate del desarrollo. Por el contrario, mi objetivo es señalar que los procesos que promueven el conflicto, las relaciones de intermediación y los accesos desiguales al interior de las redes sociales donde se mueven los actores son un reto que la creación y reproducción de capital social tiene que enfrentar en aquellas estrategias de desarrollo que pretendan influir de manera efectiva en los entornos comunitarios. 
ANEXO

\begin{tabular}{|c|c|}
\hline \multicolumn{2}{|r|}{ DEFINICIONES MÁS COMUNES DE CAPITAL SOCIAL } \\
\hline AUTOR & DEFINICIÓN \\
\hline Pierre Bourdieu & $\begin{array}{l}\text { Conjunto de recursos reales o potenciales a disposición de los } \\
\text { integrantes de una red durable de relaciones más o menos } \\
\text { institucionalizadas. }\end{array}$ \\
\hline James Coleman & $\begin{array}{l}\text { Recursos socioestructurales que constituyen un activo de capital para } \\
\text { el individuo y facilitan ciertas acciones comunes de quienes } \\
\text { conforman esa estructura. }\end{array}$ \\
\hline Robert Putnam & $\begin{array}{l}\text { Aspectos de las organizaciones sociales, tales como las redes, las } \\
\text { normas y la confianza, que facilitan la acción y la cooperación para } \\
\text { beneficio mutuo. El capital social incrementa los beneficios de la } \\
\text { inversión en capital físico y humano. }\end{array}$ \\
\hline Francis Fukuyama & $\begin{array}{l}\text { culturales que contribuyen a una } \\
\text { uncionamiento institucional. }\end{array}$ \\
\hline $\begin{array}{l}\text { Douglas North y } \\
\text { Olson }\end{array}$ & $\begin{array}{l}\text { Incorpora no sólo las relaciones informales, horizontales, locales } \\
\text { jerárquicas, sino también las relaciones formales e institucionalizadas } \\
\text { y las estructuras de gobierno, el régimen político, el estado de } \\
\text { derecho, el sistema judicial y las libertades civiles y políticas. }\end{array}$ \\
\hline John D & $\begin{array}{l}\text { do de ciertas relaciones e instituciones sociales, caracterizadas } \\
\text { tudes de confianza y por conductas sostenidas de reciprocidad } \\
\text { ración. }\end{array}$ \\
\hline Deepa Narayan & $\begin{array}{l}\text { "Capital social de lazos», que genera lazos de unión entre los } \\
\text { miembros de una misma comunidad. Se limita a contribuir al } \\
\text { bienestar de sus miembros. También define el «capital social de } \\
\text { puente», que genera sinergia entre grupos disímiles. Abre } \\
\text { oportunidades económicas a aquellos que pertenecen a los grupos } \\
\text { menos poderosos o excluidos. }\end{array}$ \\
\hline $\begin{array}{l}\text { Banco Mundial } \\
\text { (Michael } \\
\text { Woolcock, Partha } \\
\text { Dasgupta y Deepa } \\
\text { Narayan) }\end{array}$ & $\begin{array}{l}\text { Instituciones, relaciones, actitudes y valores que rigen la interacción } \\
\text { de las personas y facilitan el desarrollo económico y la democracia. }\end{array}$ \\
\hline $\begin{array}{l}\text { BID } \\
\text { Klik }\end{array}$ & $\begin{array}{l}\text { Normas y redes que facilitan la acción colectiva y contribuyen al } \\
\text { beneficio común. }\end{array}$ \\
\hline $\begin{array}{l}\text { PNUD (Norbert } \\
\text { Lechner) }\end{array}$ & $\begin{array}{l}\text { Relaciones informales de confianza y cooperación (familia, } \\
\text { vecindario, colegas); asociatividad formal en organizaciones de } \\
\text { diversos tipos; marco institucional normativo y valórico de una } \\
\text { sociedad que fomenta o inhibe las relaciones de confianza y } \\
\text { compromiso cívico. }\end{array}$ \\
\hline
\end{tabular}

Fuente: Elaboración propia con base a lo planteado por Irma Arriagada (2003). 


\section{BIBLIOGRAFÍA}

Anderson, Jeanine, 2008, «Incommensurable worlds of practice and value: a view from the shanty-towns of Lima», en Indelible inequalities: new perspectives from Latin American history, politics and culture, editado por Gootenberg, Paul y Luís Reygadas, Duke University Press, Durham y Londres, pp. 99-130,.

Arriagada, Irma, 2003, «Capital social: potencialidades y limitaciones analíticas de un concepto», en Capital social: potencialidades analíticas y metodológicas para la superación de la pobreza, compilado por Arriagada, Irma y Francisca Miranda, Comisión Económica para América Latina y el Caribe, División de Desarrollo Social, Proyecto ITA/02/049, Santiago de Chile, pp. 13-83.

Arriagada, Irma y Francisca Miranda (compiladoras), 2003, Capital social: potencialidades analíticas y metodológicas para la superación de la pobreza, Comisión Económica para América Latina y el Caribe, División de Desarrollo Social, Proyecto ITA/02/049, Santiago de Chile.

Bahamondes, Miguel, 2003, «Contradicciones del concepto "capital social”», en Capital social: potencialidades analíticas y metodológicas para la superación de la pobreza, compilado por Arriagada, Irma y Francisca Miranda, Comisión Económica para América Latina y el Caribe, División de Desarrollo Social, Proyecto ITA/02/049, Santiago de Chile, pp. 167-182.

Boisier, Sergio, 2003, «Comunicaciones al Seminario-taller El largo brazo de Descartes: usos y abusos del concepto de capital social en las propuestas de desarrollo», en Capital social: potencialidades analíticas y metodológicas para la superación de la pobreza, compilado por Arriagada, Irma y Francisca Miranda, Comisión Económica para América Latina y el Caribe, División de Desarrollo Social, Proyecto ITA/02/049, Santiago de Chile, 
pp. 63-68.

Bourdieu, Pierre, 1990, «Algunas propiedades de los campos», en Sociología y Cultura, editado por García Canclini, Néstor, Consejo Nacional para la Cultura y las Artes, México, pp. 135-141.

— 1985 , «Las formas del capital. Capital Económico, capital cultural y capital social», en Poder, derecho y clases sociales, editado por Bourdieu, Pierre, Desclée, Barcelona, pp. 131-164.

De la Peña, Guillermo, 2000, «Corrupción e informalidad», en Vicios públicos, virtudes privadas: la corrupción en México, coordinado por Lomnitz, Claudio, Centro de Investigaciones y Estudios en Antropología Social, Miguel Ángel Porrúa, México, pp. 113, 128.

Durston, John, 2001, Capital social. Parte del problema, parte de la solución. Su papel en la persistencia y en la superación de la pobreza en América Latina y el Caribe, Comisión Económica para América Latina y el Caribe, División de Desarrollo Social, Santiago de Chile.

——, 2000, ¿Qué es el capital social comunitario?, Comisión Económica para América Latina y el Caribe, División de Desarrollo Social, Santiago de Chile.

Escobar, Arturo, 1997, «Antropología y desarrollo», en Revista Internacional de Ciencias sociales, n. 154, Organización de las Naciones Unidas para la Educación, la Ciencia y la Cultura, en http://www.unesco.org/issj/rics154/escobarspa.html [consulta: 28 de octubre de 2009].

Forni, Pablo, Marcelo Siles y Lucrecia Barreiro, 2004, ¿Qué es el capital social cómo 
analizarlo en contextos de exclusión social y pobreza? Estudio de caso en Buenos Aires Argentina, Research Report \#35, The Julian Samora Research Institute, Michigan State University, East Lansing, Michigan.

Gallo, María Teresa y Rubén Garrido Yserte, 2009, El capital social. Qué es y por qué importa, Instituto Universitario de Análisis Económico y Social, Universidad de Alcalá, Madrid.

Gow, David D., 2002, «Anthropology and development: Evil twin or moral narrative?», en Human Organization, v. 61, n. 4, Society of Applied Anthropology, Washington, pp. 299313.

Granovetter, Mark S., 1973, «The strength of weak ties», en American Journal of Sociology, v. 78, n. 6, , .The University of Chicago Press, Chicago, Illinois, pp. 1360-1380.

Harriss, John y Paolo De Renzio, 1997, «An introductory bibliographic essay», en Journal of International Development, v. 9, n. 7, John Wiley \& Sons, pp. 919-937.

Jacobs, Jane, 1961, The Death and Life of Great American Cities, Random House, New York.

Kliksberg, Bernardo, 2000, «El rol del capital social y de la cultura en el proceso de desarrollo», en Capital social y cultura: claves estratégicas para el desarrollo, editado por Kliksberg, Bernardo y Luciano Tomassini, Banco Interamericano de Desarrollo, Universidad de Maryland, Fondo de Cultura Económica, Buenos Aires, pp. 19-58.

Levi, Margaret, 1996, «Social and Unsocial Capital: A Review Essay of Robert Putnam’s Making Democracy Work», en Politics \& Society, v. 24, n. 1, Mary-Ann Twist, pp. 45-55. 
Lomnitz, Larissa, 1994, Redes sociales, cultura y poder. Ensayos de antropología latinoamericana. Facultad Latinoamericana de Ciencias Sociales, Miguel Ángel Porrúa, México.

Loury, Glenn C., 1977, «A Dynamic Theory of Racial Income Differences», en Women, Minorities and Employment Discrimination, editado por Lamond, A. y P. Wallace, Lexington Books, Washington, pp. 153-186.

Marrero, Adriana, 2006, «La teoría del capital social. Una crítica en perspectiva latinoamericana», en Revista Arxius de Ciencias Sociales, n. 14, Universidad de Valencia, España, pp. 73-89.

Millán, Rene y Sara Gordon, 2004, «Capital social: una lectura de tres perspectivas clásicas», en Revista Mexicana de Sociología, año 66, n. 4, Universidad Nacional Autónoma de México, México, pp. 711-747.

Miranda, Francisca, 2003, «Conclusiones», en Capital social: potencialidades analíticas y metodológicas para la superación de la pobreza, compilado por Arriagada, Irma y Francisca Miranda, Comisión Económica para América Latina y el Caribe, División de Desarrollo Social, Proyecto ITA/02/049, Santiago de Chile, pp. 295-308.

Molina, José Luis, 2001, El análisis de las redes sociales. Una introducción, Ediciones Bellatierra, Barcelona.

Montaño, Sonia, 2003, «Aportes, sombras y ausencias en el debate sobre capital social», en Capital social: potencialidades analíticas y metodológicas para la superación de la pobreza, compilado por Arriagada, Irma y Francisca Miranda, Comisión Económica para América Latina y el Caribe, División de Desarrollo Social, Proyecto ITA/02/049, Santiago de Chile, pp. 69-77. 
Natal, Alejandro, 2010, «La sociedad civil como espacio de conflicto: El caso de Valle de Bravo», en Participación y ciudadanía en México, coordinado por Pablo Castro Domingo y Héctor Tejera Gaona, Universidad Autónoma Metropolitana, Juan Pablos Editor, México, pp. 229-263.

Portes, Alejandro, 1999, «Capital social: sus orígenes y aplicaciones en la sociología moderna», en De igual a igual. El desafío del Estado ante los nuevos problemas sociales, compilado por Carpio, Jorge e Irene Novacovsky, Fondo de Cultura Económica, Facultad Latinoamericana de Ciencias Sociales, Buenos Aires, pp. 243-266.

Putnam, Robert, 1993, Making democracy work: civic traditions in modern Italy, Princeton University Press, Princeton, N. J.

Putzel, James, 1997, «Policy arena accounting for the 'dark side' of social capital: reading Robert Putnam on democracy», en Journal of International Development, v. 9, n. 7, John Wiley \& Sons, pp. 939-949.

Rodríguez, Luís, 2010, Procesos políticos, gestión local y pluralismo cultural en el municipio de las Margaritas, Chiapas, México (1930-2005). Una perspectiva antropológica de la acción pública, Tesis de doctorado, El Colegio Mexiquense, Zinacantepec.

Rodríguez, Emanuel, 2007, Migración interna, redes sociales y capital social: la articulación de los migrantes tabasqueños en Villa Guerrero, Estado de México, Tesis de licenciatura en antropología social, Escuela Nacional de Antropología e Historia, México.

Swartz, Marc J., Victor W. Turner y Arthur Tuden, 1966, «Introduction», en Political Anthropology, editado por Swartz, Marc J., Victor W. Turner y Arthur Tuden, Aldine 
Publishing Company, Chicago, pp. 1-41. (Traducción: 1994, «Antropología política una introducción», en Alteridades, año 4, n. 8, Universidad Autónoma Metropolitana, México).

\section{NOTAS}

${ }^{1}$ El desarrollo constituye por sí mismo un concepto problemático pues siempre ha sido vinculado a un cierto grado de «intervención» en las sociedades donde se implementan los planes y programas de acción para solucionar alguna problemática específica.

${ }^{2}$ El trabajo sobre la inequidad de Glenn Loury representa un fructífero intento por agrupar el conjunto de redes de relaciones familiares y sociales que usan los jóvenes para hacer rendir su «capital humano» (Trigilia 2003:7 citado en Marrero 2006). Por otro lado, Mark Granovetter (1973) aportó elementos centrales a la discusión sobre el capital social al considerar que los actores económicos no son individuos aislados sino que están incrustados (embeddedness) en relaciones, redes y estructuras sociales.

${ }^{3}$ Bourdieu distingue entre capital económico, capital social, capital cultural y capital simbólico.

${ }^{4}$ La contextualización del análisis del capital social dentro de la teoría de los campos sociales se ha dejado de lado en los enfoques y las aplicaciones contemporáneas que analizaremos a continuación. Posteriormente debatiremos la reincorporación de este concepto a dicho entramado teórico con el fin de enriquecer sus cualidades analíticas.

${ }^{5}$ La concepción del capital social como recurso es analítica en Coleman y no se refiere a que éste puede ser propiedad privada de alguno de los actores que lo usan o lo obtienen en las redes de relaciones dado que el beneficio de la inversión no es apropiable de manera única.

${ }^{6}$ En el cuadro anexo (al final del documento) se presentan algunos de los referentes conceptuales de la amplia gama de reflexiones en el debate sobre capital social, que por supuesto exceden lo expuesto en este trabajo.

${ }^{7} \mathrm{Si}$ bien las tipologías tienen alguna utilidad como tipos ideales para saber qué elementos se asemejan a o difieren de los elementos constitutivos de cada categoría, es evidente que la construcción dinámica de las relaciones de acuerdo a cada contexto no debe de hacernos pensar linealmente que lo que se ha aplicado en una comunidad tendrá los mismos resultados en otra con similares características.

${ }^{8}$ En este punto destacan los trabajos académicos compilados por Irma Arriagada y Francisca Miranda (2003).

${ }^{9}$ Sobre las experiencias y los resultados que han tenido los proyectos aplicativos existe una amplia literatura editada por las agencias internacionales (BM, PNUD, CEPAL, etc.). Un panorama general de estos trabajos se puede consultar en María Teresa Gallo Rivera y Rubén Garrido Yserte (2009), específicamente en el capítulo 6.

${ }^{10} \mathrm{El}$ aumento de las investigaciones teóricas y empíricas, así como de programas y proyectos de intervención centradas en el concepto de capital social como eje para el desarrollo, la participación democrática y la definición de políticas públicas, aumentó de manera considerable a partir de la aparición del trabajo de C. Grootaert (1998) cuyo título no puede ser más ilustrativo Capital social: ¿el eslabón perdido? (Boisier 2003).

${ }^{11}$ En su investigación, Kliskberg (2000) analizó detalladamente el desarrollo urbanístico para personas sin vivienda «Villa El Salvador» en Perú; las «Ferias del consumo familiar» en Venezuela; y los «Presupuestos Participativos» de Brasil y Porto Alegre.

12 Ésta quizás pueda ser una de las mejores enseñanzas que han dejado (y que se pueden retomar de) las experiencias exitosas. Algunos de estos casos se pueden revisar en Bernardo Kliksberg (2000).

13 En un estudio sobre la articulación y el empoderamiento de migrantes temporales en empresas agroindustriales del estado de México, me centré en el análisis de las redes de relaciones y en el capital social de los trabajadores temporales como estrategias para fomentar su incorporación a las estructuras sociales locales, sin enfocar la atención debida a los accesos diferenciados a los recursos entre hombres y mujeres (Rodríguez 2007).

${ }^{14}$ En este tipo de relaciones, es muy difícil saber quién engaña a quién con el fin de sacar una ventaja oportunista de la situación. Además, no queda claro quién debe pagar los costos de este tipo de actos, con lo 
que no siempre se reduce la incertidumbre en las transacciones y mucho menos se aumenta la participación de los actores en las estrategias de desarrollo.

${ }^{15}$ En un estudio sobre la cultura política en diversos sectores de la ciudad de Guadalajara, Jalisco, Guillermo de la Peña (2000) determinó que la organización en redes sociales supone la presencia estratégica de intermediarios (brokers) de tiempo completo.

${ }^{16}$ A diferencia del capital social, el análisis de redes ha desarrollado todo un esquema pormenorizado a nivel metodológico para potenciar su capacidad analítica y heurística. Cabe destacar que es común encontrar estas herramientas en las investigaciones que atienden al capital social.

${ }^{17}$ Esta perspectiva nace desde la antropología para superar la visión de los estudios basados en el estructuralfuncionalismo que, para analizar el hecho político, abordaban las principales instituciones de la organización social. El modelo procesual propone que, además de atender los cuestionamientos estructurales, es necesario bajar la mirada al plano microsocial y analizar las interacciones sociales del proceso político.

18 «Hablar de capital específico significa que el capital vale en relación con un campo determinado, es decir, dentro de los límites de este campo, y que sólo se puede convertir en otra especie de capital dentro de ciertas condiciones» (Bourdieu 1990: 136).

Fecha de recepción: 17 de diciembre de 2010.

Fecha de aceptación: 30 de mayo de 2011. 